\title{
FORMULATING A TRIP PRODUCTION PREDICTION MODEL FOR THE RESIDENTIAL LAND USE IN MEDIUM SIZED TOWNS OF
} KERALA

\author{
Leena Samuel Panackel ${ }^{1}$, Padmini A.K ${ }^{2}$ \\ ${ }^{1}$ M Tech Scholar, ${ }^{2}$ Head of Department, Civil Engineering Department, Rajiv Gandhi Institute of Technology, Kerala, \\ India,leena.sam111@gmail.com,nirmalap1992@gmail.com
}

\begin{abstract}
Formulating of suitable travel demand forecasting models are the key elements for the development of a long-range transportation plan. This paper focuses its study on the formulation of a trip production model using multiple regression technique for the residential land use in medium sized towns of Kerala. The trip production model estimated the number of trips that will be produced from the residential land use of these medium sized towns. The Perinthalmanna, Tirur, and Ponnani towns of Kerala were selected as the study area based on certain criteria. The data on demographic and socio-economic characteristics these areas were collected through the administration household interviews. The quantitatively and qualitatively analysis of the results were done using the correlation and multiple regression analysis. The study showed that the regression model with the independent variables such as the percentage of automobile availability, percentage of persons employed, percentage of students and percentage of pucca type of dwelling with $R^{2}$ and Adjusted $R^{2}$ value of 0.878 and 0.859 respectively gives a better estimate of the trips produced. The model accuracy was also tested by checking the validity of the assumptions employed in the multiple regression technique. Since most of the work related to traffic and transportation planning requires an effective framework for the analysis of the present and future travel demand pattern, a model forecasting the trip produced based on the above mentioned characteristics shall be advantageous for a speedy travel demand forecast.
\end{abstract}

Index Terms: Multiple Linear Regression, Residential Land Use, Socio-Economic Characteristics, Trip Production $* * *$

\section{INTRODUCTION}

Transportation forms the backbone of a town's development. There are no such things as town without traffic and human settlement grow up at every transportation node. It is a known fact that towns determine the axis along which traffic must move and that transportation gives any town its form and confirms the layout. Transportation is known to be the movement of people, goods and service from one place to another under a desirable condition while transportation planning is concerned with the development of a plan with respect to the social, economic and environmental impacts of the populace to enhance positive goals [1]. The fundamental goal of transportation planning is to accommodate the need for mobility in order to provide efficient access to various activities that satisfy human needs. The traffic demands are usually forecasted based on the assumption that they are related to human travel behavior, land use, and travel patterns. Transportation planning also includes the travel demand and travel trip alongside with its generation, distribution, mode of travel and route assignment. Each trip is made for a particular purpose and is also dependent on many factors varying from income, automobile availability, age, distance and many more [2].
The history of demand modeling for person travel has been dominated by the modeling approach that has come to be referred to as the four step model [3]. The urban travel estimation process which determines both present and future travel patterns are Trip Generation, Trip Distribution, Modal Split and Traffic Assignment. The number of trips produced is one of the most vital tools for the future planning of the transportation networks and for the proper land use allocation. Thus, this research will focus on the trip production in the study location.

Trip generation is the first step in the conventional four step transportation forecasting process, which is widely used for forecasting travel demands. It predicts the number of trips originating in or destined for a particular traffic analysis zone [3-4].The trip generation consist of the trip production and trip attraction. The trip production analysis focusing on residences and residential trip generation is thought of as a function of social and economic attributes of households [1-5]. At the level of the traffic analysis zone, residential land uses produce or generate trips. Residential land use is the main trip generator in an urban context. Household generated trips comprise a major portion of all trips in an urban area. Actually more than $80 \%$ of trips in an urban area are generated by the 
residents of households in the area. The various characteristics of residential land use such as density, demographic, socioeconomic factors etc influence the number of trip generated from an area. The household surveys are conducted to obtain the socio-economic as well as trip details of the residential areas [5-6]. Since most of the work related to traffic and transportation planning requires an effective framework for the analysis of the present and future travel demand pattern, a model forecasting the trip production based on the above mentioned characteristics shall be advantageous for a speedy travel demand forecast. The majority of trip-generation studies performed have used multiple regression analysis to develop the prediction equations for the trips generated by various types of land use.

The various papers that based their studies on the forecasting of trip generation were generally seen to use certain similar parameters in their work. It was mainly seen that most these authors used the explanatory variables such as socio-economic data, demographic details of household and land use pattern has the basic and key input data in their studies [1-6]. The most popular methods have come to be known as Land Area Trip Rate Analysis, Cross Classification Analysis, and Regression Analysis [8-9]. Many of the studies have relied upon on the multiple regression technique for determining the gross number of trips produced from the study areas [1-7]. The attempt of using this method even at the recent times gives a strong indication of the reliability of this method in forecasting trip production. But at the same time, some of the papers have tried to stand out by implementing method different from the conventional trip generation forecasting method [10-12]. These papers have tried to improve the existing techniques through their proposals.

Thus in this paper the main technique used for the trip production analysis is the multiple linear regression method. This process consists of developing equations in which trips are related to independent variables which explain the variation in dependent variables (trips). The equations are usually developed based on data aggregated to the zone level observation. The correlation and multiple regression analysis was used for the analysis work in this paper. Thus as an output the model would be able to forecast the future trips produced from residential land use of medium size towns throughout Kerala.

\section{STUDY AREA}

The selection of the study area forms the first and most primary step towards a model building. The study area for this report is selected in such a way that they represent the majority of the towns in Kerala and thus expanding the scope of the model and not restricting it to a certain area.

\subsection{Selection Criteria}

The selection of the towns to represent the study area was chosen in such a way as to satisfy three criteria. The towns in Kerala can be broadly classified into three types such as coastal, midland and highland area based on its geographical division, most of the towns are of medium sized and they are of varied characteristics. Thus taking into account these three criteria, the Perinthalmanna, Tirur, and Ponnani towns in the Malappuram district were selected as the study area. Perinthalmanna is a town with strong historical and cultural heritage with an area of $34.41 \mathrm{~km} 2$ and constitutes of 34 wards. It has for last several centuries remained as the center of trading and commercial activity for several villages around it and as of 2001census, Perinthalmanna had a population of 44,613. Tirur is a municipal town in Malappuram district spread over an area of $16.55 \mathrm{~km} 2$ and constitutes of 38 wards. It is one of the most important business centers of Malappuram district and has a population of 53,650. Ponnani is a coastal municipality and an important fishing center in Malappuram district spread over an area of $24.82 \mathrm{~km} 2$ and constitutes of 51 wards and has a population of 87,356 . The Perinthalmanna and Tirur town belongs to the geographical classification of midland area, whereas the Ponnani town falls under the coastal classification and all these towns are of medium sized. The Perinthalmanna town is known as the hospital city, the Tirur town is known for its commercial development whereas the Ponnani town is known as important fishing center. Since they satisfy the three stated criteria, these towns were taken as the study area.

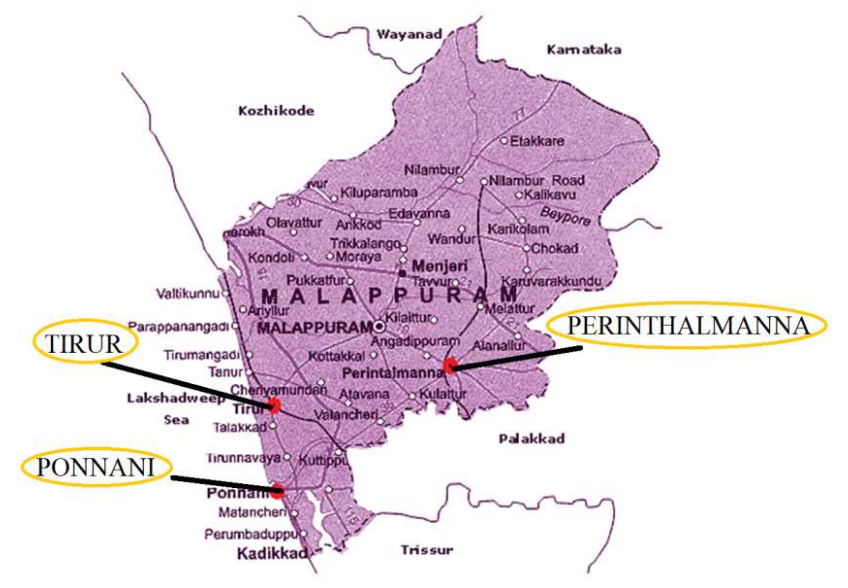

Fig -1: Location of selected study area

\section{METHODOLOGY}

The goal of trip production process is to forecast the number of person-trips that will begin from the residential land use contained in the area of study. The study area were selected such that they satisfy three criteria that they belong to a geographical division of coastal, midland and highland area, 
the towns are of medium size and are of varied characteristics to represent the majority of towns in Kerala. The work is conducted by dividing the towns into zones and for the analysis work, each of the zones of these towns shall coincide with the municipal wards consisting of low, medium and high residential area. The work is progressed by the collection of relevant land use characteristics of the selected towns in Kerala, for obtaining the concentration of residential land use. The research on forecasting trip production is carried out by selecting 50 wards from the selected towns on the basis of the concentration index of the residential land use. Household survey was conducted through the administration of questionnaires. The socio-economic and trip details pertaining to the selected wards were collected. The socio-economic characteristics that are relevant for this study can be broadly classified as household size, age, income, vehicle ownership and other home-based elements. The origin-destination details obtained from the household survey was used to form the O-D matrix that gives the gross number of trips produced from each of the wards within the particular zones. The multiple linear regression technique was implemented for the model development. The sample size for the model formulation and validation was selected using the holdout method, where $60 \%$ of the data was used as the training set and $40 \%$ of data as the testing set. The statistical analysis of the data was done using the correlation and multiple regression analysis. Thus as an output the model would be able to forecast the future trips produced from residential land use of medium sized towns throughout Kerala.

\section{DATA COLLECTION}

\subsection{Dependent Variables}

The dependent variables would be the number of trip produced from each of the wards within the divided zones in the selected towns. The data pertaining to the number of trips produced from each of the ward was obtained by conducting the socio-economic survey and forming the O-D matrix. To understand the behavior and factors affecting the travel, one has got the origin of travel when the decision for travel is made. It is where people live as family which is the household. Therefore household data is considered to be the most basic and authentic information about the travel pattern of a city. Ideally one should take the details of all the people in the study to get complete travel details. However, this is not feasible due to large requirement of time and resources needed. In addition this will cause difficulties in handling these large data in modeling stage. Therefore, some sample households are randomly selected and survey is conducted to get the household data. Higher sample size is required for large population size, and vice-versa. Normally minimum ten percent samples are required for population less than 50,000. The socio-economic survey for the thesis work was carried out by using the sampling technique, as it is not possible to take into account the entire households within the town. Thus a sampling of $10 \%$ was taken to conduct the socio-economic survey. From the socio-economic survey, the origin destination details of each ward within the zones were obtained and these trip details were used to form the O-D matrix. The chart 1 shows the number of trips produced from each of the selected wards within the three selected towns.

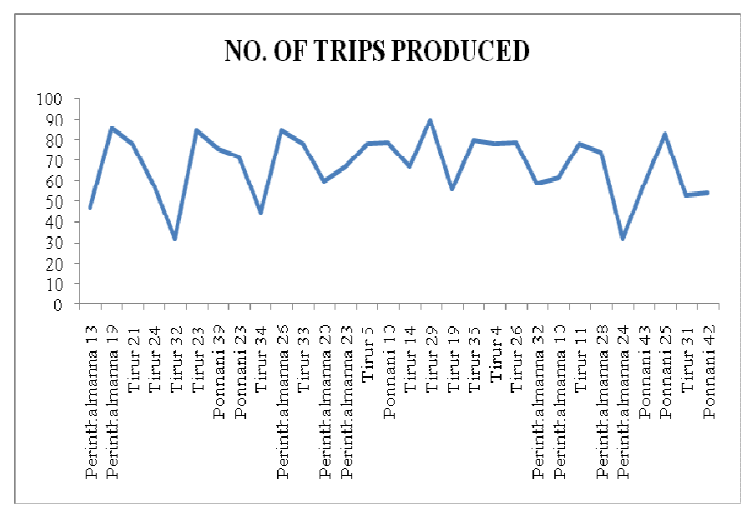

Chart -1: Trips produced from the selected wards

\subsection{Explanatory Variables}

The independent or explanatory variables are key input factors that influence the number of trips produced from the wards of a town. The identified independent variables are listed below:

i. Income

ii. Automobile availability

iii. Dwelling type

iv. Average household size

v. Age

vi. Gender

vii. Students

viii. Persons employed

ix. Marital status

These variables were identified based on the review of several related papers and also based on the influence of these variables on the trip produced. The socio economic survey was conducted to obtain information regarding the above identified variables. For conducting of the socio-economic survey, the key factor is the modulation of an ideal survey questionnaire. From the survey, the details pertaining to the identified independent variables are collected for the 30 wards that was used in the model formulation part and for the 20 wards that was used for the model validation part.

\section{STATISTICAL ANALYSIS OF DATA}

The majority of trip-generation studies performed have used multiple regression analysis to develop the prediction equations for the trips generated by various types of land use. Multiple linear regression attempts to model the relationship between two or more explanatory variables and a response variable by fitting a linear equation to observed data. 


\subsection{Model Formulation}

Formulating good models is an art bordering on a science. The artistic ability is in developing simple models that are nevertheless good approximations of reality. In this paper, 30 wards along with its identified independent variables were selected for the model formulation. Correlation and regression analysis was performed using Microsoft excel for model formulation.

\subsubsection{Correlation of Variables}

Correlation and regression analysis are related in the sense that both deal with relationships among variables. The correlation coefficient is a measure of linear association between two variables. Values of the correlation coefficient are always between -1 and +1 . A correlation coefficient of +1 indicates that two variables are perfectly related in a positive linear sense, a correlation coefficient of -1 indicates that two variables are perfectly related in a negative linear sense, and a correlation coefficient of 0 indicates that there is no linear relationship between the two variables. The table I depict the correlation between each of the independent variables with the dependent variable (trips produced). It is seen from the table I that the variables $\mathrm{x} 1, \mathrm{x} 4, \mathrm{x} 5, \mathrm{x} 12, \mathrm{x} 13, \mathrm{x} 14, \mathrm{x} 17$, and $\mathrm{x} 18$ are moderately correlated with the trip produced. Among the moderately correlated variables, the independent variables $\mathrm{x} 1$ and $\mathrm{x} 18$ are negatively correlated and $\mathrm{x} 4, \mathrm{x} 5, \mathrm{x} 12, \mathrm{x} 13, \mathrm{x} 14$, and $\mathrm{x} 17$ are positively correlated with the depended variable $\mathrm{y}$.

\subsubsection{Multicollinearity}

It occurs when there is a linear relationship among one or more of the independent variables. If one of the independent variable is regressed on the other independent variables and the resulting $R^{2}=1.0$, then the matrix of intercorrelations among the independent variables is singular and there exists no unique solution for the regression coefficients. The presence of multicollinearity makes the regression estimates unstable. A correlation matrix shows the correlation between each pair of variables. The diagonal of the matrix has values of 1 because a variable always has a perfect correlation. The magnitude of the correlation coefficient determines the strength of the correlation. The guideline below helps to determine the type of correlation between the variables:

$0<|\mathrm{r}|<0.3$ weak correlation

$0.3<|\mathrm{r}|<0.7$ moderate correlation

$|\mathrm{r}|>0.7$ strong correlation

It can be seen from the Table 2, that correlation between the selected explanatory variables are only moderate correlation.

So in order to offset the error due to multicollinearity, it is always better to avoid the combination of the independent variables having high correlation value.

Thus taking into account the collinearity coefficient of the dependent variable with the independent variable and also the presence of multicollinearity, eight explanatory variables were selected. Only the significant and moderately correlated explanatory variables were selected for the model formulation. The selected explanatory variables are listed below:

Table -1: Correlation Coefficients

\begin{tabular}{|l|l|l|}
\hline Sl No. & Independent variables & Correlation Coefficient ( r) \\
\hline 1 & Income between 0- <1000 ( x1) & -0.395 \\
\hline 2 & Income between 1000- 3000 (x2) & -0.184 \\
\hline 3 & Income between 3000-8000 ( x3) & 0.256 \\
\hline 4 & Income greater than 8000 ( x4) & 0.341 \\
\hline 5 & Average household size ( x5) & 0.456 \\
\hline 6 & Percentage of persons below 5 yrs ( x6) & -0.011 \\
\hline 7 & Percentage of persons between 5 to 20 yrs ( x7) & -0.125 \\
\hline 8 & Percentage of persons between 21 to 50 yrs ( x8) & -0.085 \\
\hline 9 & Percentage of persons above 50 yrs ( x9) & -0.226 \\
\hline 10 & Percentage of Males ( x10) & -0.077 \\
\hline 11 & Percentage of Females (x11) & 0.077 \\
\hline 12 & Percentage of automobile availability (x12) & 0.489 \\
\hline 13 & Percentage of persons employed (x13) & 0.763 \\
\hline 14 & Percentage of students ( x14) & 0.718 \\
\hline 15 & Percentage of married people (x15) & 0.194 \\
\hline 16 & Percentage of single persons ( x16) & -0.213 \\
\hline 17 & Percentage of pucca type of dwelling ( x17) & 0.548 \\
\hline 18 & Percentage of medium type of dwelling ( x18) & -0.55 \\
\hline
\end{tabular}


Table -2: Correlation Matrix of Selected Explanatory Variables

\begin{tabular}{|c|c|c|c|c|c|c|c|c|c|}
\hline & $\mathrm{y}$ & $\mathrm{x}_{1}$ & $\mathrm{x}_{4}$ & $\mathrm{x}_{5}$ & $\mathrm{x}_{12}$ & $\mathrm{x}_{13}$ & $\mathrm{x}_{14}$ & $\mathrm{x}_{17}$ & $\mathrm{x}_{18}$ \\
\hline $\mathrm{y}$ & 1 & & & & & & & & \\
\hline $\mathrm{x}_{1}$ & -0.395 & 1 & & & & & & & \\
\hline $\mathrm{x}_{4}$ & 0.341 & -0.468 & 1 & & & & & & \\
\hline $\mathrm{x}_{5}$ & 0.456 & 0.157 & -0.12 & 1 & & & & & \\
\hline $\mathrm{x}_{12}$ & 0.489 & -0.219 & 0.429 & 0.248 & 1 & & & & \\
\hline $\mathrm{x}_{13}$ & 0.763 & -0.129 & 0.07 & 0.507 & 0.184 & 1 & & & \\
\hline $\mathrm{x}_{14}$ & 0.718 & -0.198 & 0.048 & 0.458 & 0.155 & 0.524 & 1 & & \\
\hline $\mathrm{x}_{17}$ & 0.548 & -0.47 & 0.469 & 0.047 & 0.352 & 0.169 & 0.381 & 1 & \\
\hline $\mathrm{x}_{18}$ & -0.55 & 0.47 & -0.47 & -0.05 & -0.36 & -0.17 & -0.376 & -0.999 & 1 \\
\hline
\end{tabular}

- Income between $0-<1000\left(\mathrm{x}_{1}\right)$

- Income greater than $8000\left(\mathrm{x}_{4}\right)$

- Average household size $\left(\mathrm{x}_{5}\right)$

- Percentage of automobile availability $\left(\mathrm{x}_{12}\right)$

- Percentage of persons employed $\left(\mathrm{x}_{13}\right)$

- Percentage of students $\left(\mathrm{x}_{14}\right)$

- Percentage of pucca type of dwelling $\left(\mathrm{x}_{17}\right)$

- Percentage of medium type of dwelling $\left(\mathrm{x}_{18}\right)$

\subsubsection{Developing the Multiple Regression Equation}

The statistical technique employed for finding the best regression estimates was the backward elimination method. The multiple linear regression analysis of the independent variables were performed using the Microsoft excel. Using this tool, different combinations of the independent variables were regressed to find out the most accurate and suitable regression model. The regression model having highest $\mathrm{R}, \mathrm{R} 2$ and Adjusted R2 value, minimum standard error of estimate, low significance $F$ value and low $p$ value for the coefficients of independent variables and y intercept was selected as the best model using the regression analysis. The regression model consisting of the four explanatory variables that is percentage of automobile availability, percentage of persons employed, percentage of students and percentage of pucca type of dwelling was found as the most suitable regression model.

The predictive equation from the multiple regression model is

$$
\begin{gathered}
y=-28.027+\left(0.277 \times x_{12}\right)+\left(1.592 \times x_{13}\right)+\left(1.447 \times x_{14}\right)+ \\
\left(0.179 \times x_{17}\right)
\end{gathered}
$$

Where,

$\mathrm{y}=$ Trips produced from the residential areas in the medium sized towns on Kerala

$\mathrm{x}_{12}=$ Percentage of automobile availability

$\mathrm{x}_{13}=$ Percentage of persons employed

$\mathrm{x}_{14}=$ Percentage of students

$\mathrm{x}_{17}=$ Percentage of pucca type of dwelling
The first term in the prediction equation (-28.027) is a constant that represents the predicted criterion value when both predictors equal zero. The values of $0.277,1.592,1.447$ and 0.179 represent regression weights or regression coefficients.

Trip will increase on an average by $0.277,1.592,1.447$ and 0.179 trips per day for each $1 \%$ increase in the Percentage of automobile availability, Percentage of persons employed, Percentage of students and Percentage of pucca type of dwelling respectively.

The summary output of the selected regression model is given in the Table 3, 4, and 5. In the formulated trip production model regression statistics, the value $\mathrm{R}$ is a measure of the correlation between the observed value the predicted value of the criterion variable. For this model it has a correlation of 0.9372 . This shows that there is a high correlation between the observed and predicted value.

The coefficient of determination is the square of the correlation coefficient $\left(\mathrm{R}^{2}\right)$. The $\mathrm{R}$-squared of the regression is the fraction of the variation in the dependent variable that is accounted for (or predicted) by the independent variables. The obtained coefficient of determination $\left(\mathrm{R}^{2}\right)$ value is 0.8784 , which denotes that $87.84 \%$ of the trip produced is influenced by the variation in the percentage of automobile availability, percentage of persons employed, and percentage of students and by the percentage of pucca type of dwelling while $12.16 \%$ is explained by other factors. 
Adjusted R Square value takes into account the number of variables in the model and the number of observations our model is based on. This Adjusted R Square value gives the most useful measure of the success of the model. The obtained adjusted $\mathrm{R}^{2}$ value is 0.8590 , which means that $85.9 \%$ of the trip produced are influenced by the percentage of automobile availability, percentage of persons employed, percentage of students and by the percentage of pucca type of dwelling and these are the independent variable that affect the trips produced to the greatest extend or is the most significant variable when compared to the rest.

The standard error of the model, which is the standard deviation of residuals, indicates the degree of variation on the data about the regression line established that is, the error we would expect between the predicted and actual dependent variable. The standard error of the regression model is 5.8842 . This means that, the expected error for trip production predicted is off by 5.8842 trips. The error was a comparatively small when the sample size was considered.

From the Table 4 the Significance of $F$ value indicates the probability that the Regression output could have been obtained by chance. A small Significance of F confirms the validity of the Regression output. Since the obtained significance of $\mathrm{F}$ value was $4.36139 \mathrm{E}-11$, it means that there was only a $4.36139 \mathrm{E}-09 \%$ chance that the regression output was merely a chance occurrence. Thus increasing the overall accuracy and significance of the formulated model.

The p-value is a percentage and it tells you how likely it is that the coefficient for that independent variable emerged by chance and does not describe a real relationship. A p-value of 0.05 means that there is a $5 \%$ chance that the relationship emerged randomly and a $95 \%$ chance that the relationship is real. It is generally accepted practice to consider variables with a p-value of less than 0.1 as significant, though the only basis for this cutoff is convention. The lower the p-Value, the higher the likelihood that that coefficient or Y-Intercept is valid. From the Table V the p-value for the coefficient of percentage of automobile availability, percentage of persons employed percentage of students and percentage of pucca type of dwelling was $0.001,0.002,0.0000021,0.001$ and 0.004 respectively. This indicates that there was only a minor chance that the result occurred by chance. Hence the accuracy of the model increases to a greater percentage.

Table -3: Regression Statistics

\begin{tabular}{|l|c|}
\hline Multiple R & 0.9372 \\
\hline R Square & 0.8784 \\
\hline Adjusted R Square & 0.859 \\
\hline Standard Error & 5.8842 \\
\hline Observations & 30 \\
\hline
\end{tabular}

Table -4: ANOVA Test Results

\begin{tabular}{|l|c|c|c|c|c|}
\hline & $d f$ & $S S$ & $M S$ & $F$ & Significance $F$ \\
\hline Regression & 4 & 6253.38 & 1563.3 & 45.153 & $4.36 \mathrm{E}-11$ \\
\hline Residual & 25 & 865.59 & 34.623 & & \\
\hline Total & 29 & 7118.97 & & & \\
\hline
\end{tabular}

Table -5: Regression Coefficients

\begin{tabular}{|c|c|c|c|c|c|c|}
\hline & Coefficients & Standard Error & tStat & P-value & Lower 95\% & Upper 95\% \\
\hline Intercept & -27.456837 & 7.5192 & -3.652 & 0.001264 & -42.976 & -11.938 \\
\hline $\mathrm{x}_{4}$ & 0.247103 & 0.2294 & 1.077 & 0.292103 & -0.226 & 0.721 \\
\hline $\mathrm{x}_{12}$ & 0.247566 & 0.0846 & 2.926 & 0.007396 & 0.073 & 0.422 \\
\hline $\mathrm{x}_{13}$ & 1.58137 & 0.2589 & 6.109 & 0.000003 & 1.047 & 2.116 \\
\hline $\mathrm{x}_{14}$ & 1.522394 & 0.4056 & 3.754 & 0.00098 & 0.685 & 2.359 \\
\hline $\mathrm{x}_{17}$ & 0.151125 & 0.0624 & 2.422 & 0.023348 & 0.022 & 0.28 \\
\hline
\end{tabular}

\subsubsection{Examining the Regression Models Assumptions}

As with all statistical procedures, multiple linear regression analysis rests on basic assumptions about the population from where the data have been derived. The results of the analysis are only reliable when these assumptions are satisfied. These assumptions are:

- Linearity - the relationship between the predictors and the outcome variable should be linear. In other words, each increase by one unit in an explanatory variable is associated with a fixed increase in the outcome variable.

- Normality - the errors in the conditional distributions should be normally distributed. The individual data points of Y (the response variable) for each of the explanatory variables are normally distributed about the line of means (regression line).

- Homoscedasticity - the errors in the conditional distributions should have constant variance. The variance of the data points about the line of means is the same for each explanatory variable.

- Independence - the errors in the conditional distributions are correlated. The explanatory variables are independent of each other i.e. knowing the value of one or more of the independent variables do not tell us anything about the others.

- Muliticollinearity

- Outliers- An outlier is an extreme observation. Typically points further than, say, three or four standard deviations from the mean are considered as "outliers".

Diagnostic procedures are intended to check how well the assumptions of multiple linear regression are satisfied. 
1. Check for Linearity

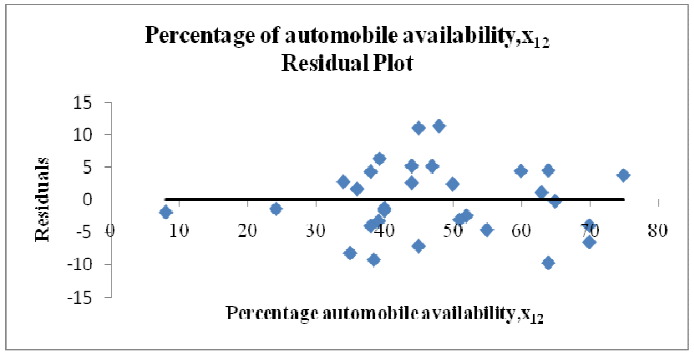

Fig -2: Residual plot of \% automobile availability

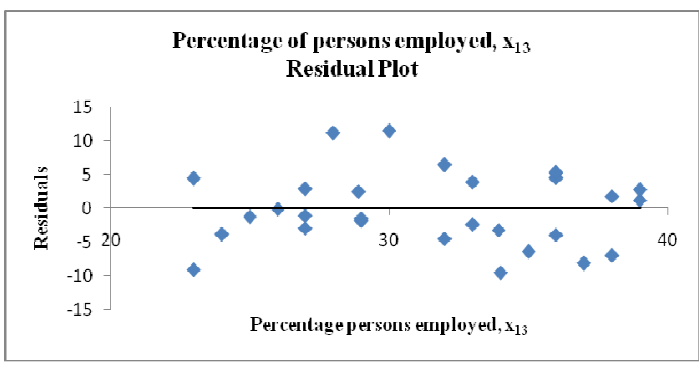

Fig -3: Residual plot of \% persons employed

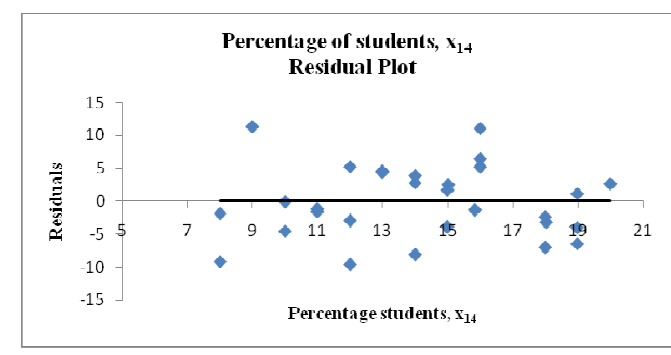

Fig -4: Residual plot of \% students

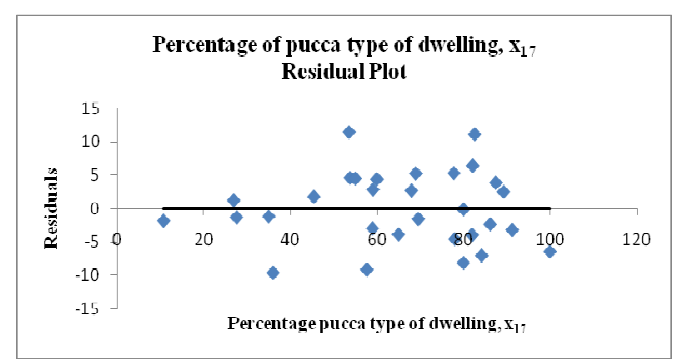

Fig -5: Residual plot of \% of pucca type of dwelling

It can be clearly seen from the residual plots of the residual vs. independent variables that the dependent and independent variables are linearly related. The plots points almost lie within the horizontal lie centering around the zero axis. The points are symmetrical around the horizontal line.
2. Check for Normality

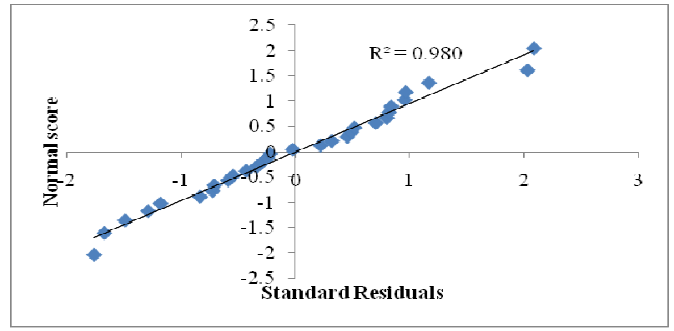

Fig -6: Normal Probability Plot

It is evident from the normal probability plot given above that the points show least amount of deviation from the normal distribution and the points on this plot falls close to the diagonal line. Thus the assumption of the selected regression model be normally distributed is satisfied.

3. Check for independence

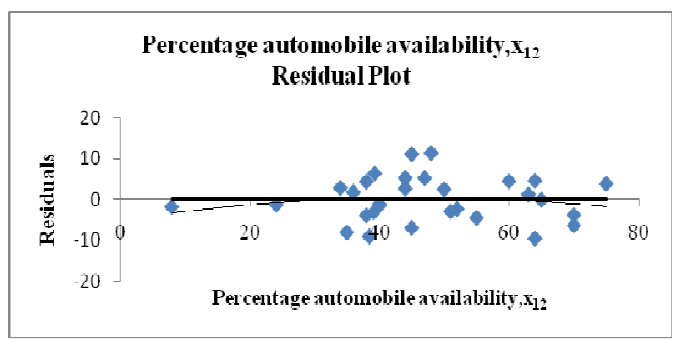

Fig -7: Residual plot of \% automobile availability

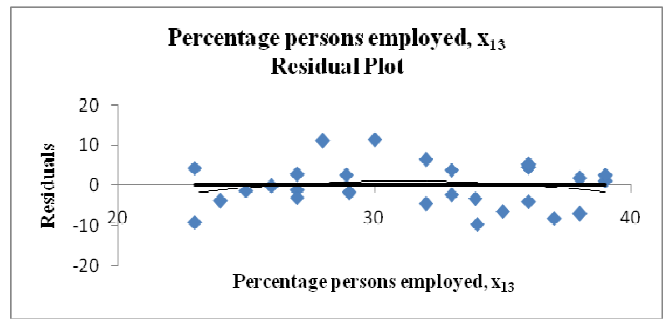

Fig -8: Residual Plot of \% Persons Employed

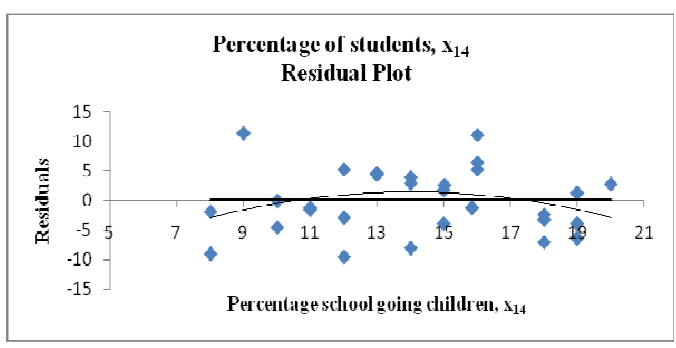

Fig -9: Residual plot of \% of students 


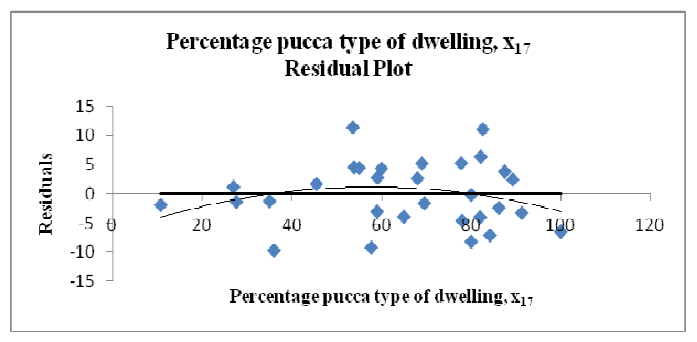

Fig -10: Residual plot of \% of pucca type of dwelling

It can be seen from the plots that the residual plot indicates a slight upward curvature. But since the curvature is only minor, it is not considered significant and thus the assumption of the model to be independent is satisfied.

\section{Check for Homogeneous Variance}

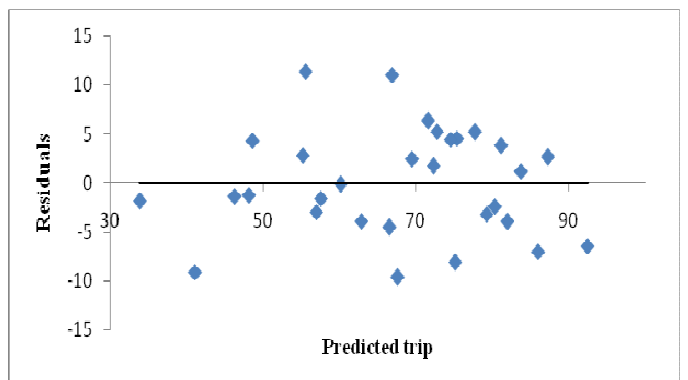

Fig -11: Residual plot of the residuals versus the predicted values of the dependent variables.

It can be seen that the residuals are more or less evenly spread out in a random manner along the horizontal line. Thus the assumption of homogeneous variance is also satisfied, thereby increasing the validity of the proposed regression model.

5. Check for Multicollinearity

Table -6: Detection of Multicollinearity

\begin{tabular}{|c|c|c|c|c|}
\hline & $\mathrm{x}_{12}$ & $\mathrm{x}_{13}$ & $\mathrm{x}_{14}$ & $\mathrm{x}_{17}$ \\
\hline $\mathrm{x}_{12}$ & 1 & & & \\
\hline $\mathrm{x}_{13}$ & 0.18 & 1 & & \\
\hline $\mathrm{x}_{14}$ & 0.15 & 0.52 & 1 & \\
\hline $\mathrm{x}_{17}$ & 0.35 & 0.17 & 0.38 & 1 \\
\hline
\end{tabular}

It can be seen from the correlation matrix that the dependency of the independent variables is very less, except for the correlation between the percentage of persons employed and the percentage students. But since it is only 0.52, we can combine this combination in the model equation without affecting the accuracy of the model.
6. Presence of outlier

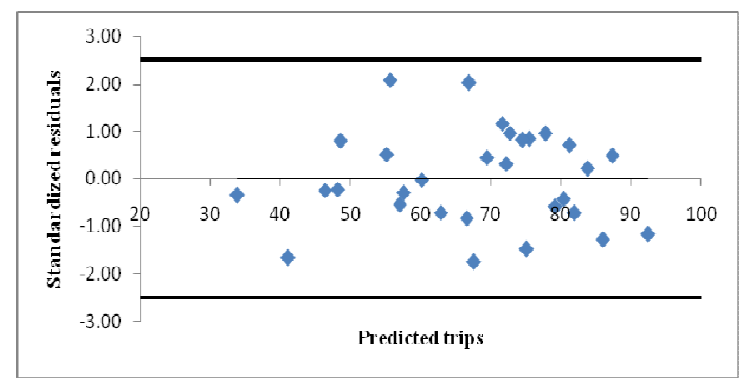

Fig -12: Standardized Residuals versus Fitted Value

Two horizontal lines corresponding to residual values of +2.5 and -2.5 are useful to distinguish between small and large residuals. Since the residual lie within the horizontal band, we can conclude that the leverage is good or the assumption is satisfied.

Thus the above 6 assumptions of multiple regression analysis is satisfied by the formulated regression model and this means that all the information available from the pattern is used. Thus it indicates the strong validity of the formulated regression model

\section{CONCLUSIONS AND RECOMMENDATION}

In this study a trip production model was developed using the multiple linear regression analysis. This regression model would be able to forecast the number of trips produced from the residential land use in medium sized towns of Kerala. The selection of the study area for the model development was such that they satisfy certain criteria so as to represent the majority of towns in Kerala. Several related studies have highlighted the influence of a number of independent variables on the trips produced. The selection of the independent variable for the study was based on the review of these studies and also based on its influence on the dependent variable. From regression analysis it was found that certain explanatory variables were found to have a greater influence on the trips produced from the residential land use when compared to the others. Also the accuracy of the formulated regression model was proved by checking the validity of the assumptions taken in the multiple regression method.

The significance of this paper lies in the fact that many of the developed trip production models of the past studies have a great dependency on the land bearings and local conditions of the study area and thus limiting its applications in all places. A model forecasting the trips produced from the residential land use of medium sized towns in Kerala would be great aid in the transportation network planning, as not many prominent studies as been conducted in Kerala relating to the forecasting of trip productions based on the residential land use. This study as also tried to include a number of varied independent 
variables and analyze their effect on the dependent variable. Due to time and cost constraints, only a certain percentage of the data was collected as the sample. Another limitation is the assumptions in the multiple regression analysis. Out of the results and data analysis of this study it can be recommended that the further accuracy of the model can be obtained by increasing the sample size used for the model development. The inclusion of more number of independent variables would also aid in the further research of their effect on the number of trips produced from a residential land use.

\section{ACKNOWLEDGEMENTS}

The authors are grateful to many persons who provided assistance with this paper and the study upon which it draws. We are thankful to the staff of the Town and Country Planning Department, Malappuram, for their contributions to this study. We would also like to extend our sincere thanks to the faculty of Department of Civil Engineering at Rajiv Institute of Technology, Kottayam for their valuable support during the making of this paper.

\section{REFERENCES}

[1] Olugbenga Joseph and Oluyemisi Opeyemi, "Regression Model of Household Trip Generation of Ado-Ekiti Township in Nigeria," European Journal of Scientific Research, ISSN 1450-216X Vol.28, no.1, EuroJournals Publishing, Inc. 2009, , pp.132-140.

[2] William J. Fogarty, "Trip Production Forecasting Models for Urban Areas," Transportation Engineering Journal () ASCE, Vol. 102, No. 4, November 1976, pp. 831-845.

[3] Michael G. McNally, "The Four Step Model," Institute of Transportation Studies University of California, 2007.

[4] Kevin B. Modi, L. B. Zala, T. A. Desai, and F. S. Umrigar, "Transportation Planning Models," National Conference on Recent Trends in Engineering \& Technology, May 2011.

[5] Charles L. Purvis, Miguel Iglesias, and Victoria A. Eisen, "Incorporating Work Trip Accessibility in Non-Work Trip Generation Models in the San Francisco Bay Area," Paper submitted to the Transportation Research Board for presentation at the 75th Annual Meeting, January 1996.

[6] Nonito M. Magdayojr, "Study on the Application of Trip Generation Analysis for Residential Condominium Developments in Metro Manila," Final Paper, Undergraduate Research Program in Civil Engineering, march 2008

[7] John S. Miller, P.E.Lester A. Hoel, P.E. Arkopal K. Goswami, and Jared M. Ulmer, "Borrowing Residential Trip Generation Rates," Journal of Tansportation Engineering (C) ASCE, February 2006, pp. 105-113.

[8] Papacostas C.S. and Prevedouros P.D., "Transportation Engineering and Planning," SI Edition, Prentice-Hall Inc Singapore, 2005.

[9] Abdul Khalik Al-Taei and Amal M. Taher, "Prediction Analysis of Trip Production Using Cross-Classification Technique,” Al-Rafidain Engineering, vol.14, no.4, 2006.
[10] Matthew Femal, "Improving Trip Generation Equations," Thesis report, School of Engineering and Applied Science, University of Virginia, 2010.

[11] Michael Anderson and Justin P. Olander, "Evaluation of Two Trip Generation Techniques for Small Area Travel Models," Journal of Urban Planning and Development (C) ASCE, vol. 128, no. 2, June 2002, pp. 77-88.

[12]Valerian Kwigizile and Hualiang HarryTeng, "Comparison of Methods for Defining Geographical Connectivity for Variables of Trip Generation Models" ,Journal of Transportation Engineering (C) ASCE, vol. 135, no. 7, July 2009, pp. 454-466.

\section{BIOGRAPHIES}

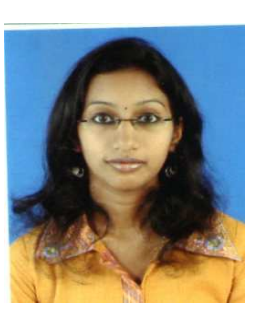

Leena Samuel Panackel has obtained her B.Tech in Civil Engineering from Cochin University of Science and Technology (CUSAT), Kerala. Presently she is pursuing her M.Tech from Rajiv Gandhi Institute of Technology (RIT),Kottaya. her area of research is transportation planning

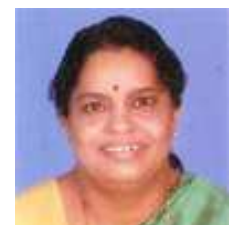

Dr. Padmini A.K. is working as Professor and HOD in the Department of Civil Engineering at Rajiv Gandhi Institute of Technology (RIT), Kottayam, Kerala. She has obtained her B.Tech in Civil Engineering from the Calicut University. She received her M.Tech and $\mathrm{PhD}$ degree from IIT, Madras. She has published several papers in various reputed Journals and National Conferences. She has experience in research and development from 1996. She has life membership of ICI and ISTE .She has a teaching experience of 27 years in various reputed Engineering Colleges. Her area of research includes durable construction and transportation planning. 